\title{
Decays of neutral pions: Electromagnetic transition form factor and radiative corrections
}

\author{
Tomáš Husek ${ }^{1, a}$ \\ ${ }^{1}$ Charles University, Prague, Czech Republic
}

\begin{abstract}
We briefly summarize experimental and theoretical results on the rare decay $\pi^{0} \rightarrow e^{+} e^{-}$. The notorious $3.3 \sigma$ discrepancy between the Standard Model prediction and the experimental value provided by $\mathrm{KTeV}$ collaboration is discussed in the view of a complete set of next-to-leading-order QED radiative corrections. We also present the Two-Hadron Saturation (THS) scenario for the $P V V$ correlator and apply it to the decay under discussion. The discrepancy under discussion then reduces down to $1.8 \sigma$. Finally, we turn our attention the the Dalitz decay $\pi^{0} \rightarrow e^{+} e^{-} \gamma$. We have recalculated the Mikaelian and Smith radiative corrections beyond the soft-photon approximation, i.e. over the whole range of the Dalitz plot and with no restrictions on the radiative photon. In contrast to the previous calculations, we also included the one-photon irreducible contribution at one-loop level. Our results can be also used for a further treatment of the processes with heavier particles in the final state.
\end{abstract}

\section{Introduction}

Discovering and quantifying beyond-Standard Model phenomena at the precision frontier is a very complicated task, since it goes along with having first our present knowledge under control. The appropriate theoretical prediction shall provide sufficiently low uncertainties together with its predictions such that the eventual disagreement is clearly and correctly revealed.

Besides the well-known discrepancy between the measurement of the anomalous magnetic moment of the muon and the theoretical prediction based on the Standard Model (SM), one of the other examples of such disagreement is the rare decay of a neutral pion, i.e. the process $\pi^{0} \rightarrow e^{+} e^{-}$. The precise measurement of its branching ratio was done by $\mathrm{KTeV}$ experiment at Fermilab [1] with the result

$$
B^{\mathrm{KTeV}}\left(\pi^{0} \rightarrow e^{+} e^{-}(\gamma), x_{\mathrm{D}}>0.95\right)=(6.44 \pm 0.25 \pm 0.22) \times 10^{-8} .
$$

Soon after it has been shown that the prediction based on the Standard Model is in the $3.3 \sigma$ disagreement with this measurement [2], which immediately drew attention of the theorists for the following years. One can immediately think about the possibility of this being a sign for new physics. But before drawing firm conclusions of this kind, the strategy should be first to look properly for a more conventional solution within the Standard Model. In what follows we will investigate in detail the radiative corrections and suggest a new model for the electromagnetic transition form factor of a neutral pion.

\footnotetext{
ae-mail: husek@ipnp.mff.cuni.cz
} 


\section{Leading order}

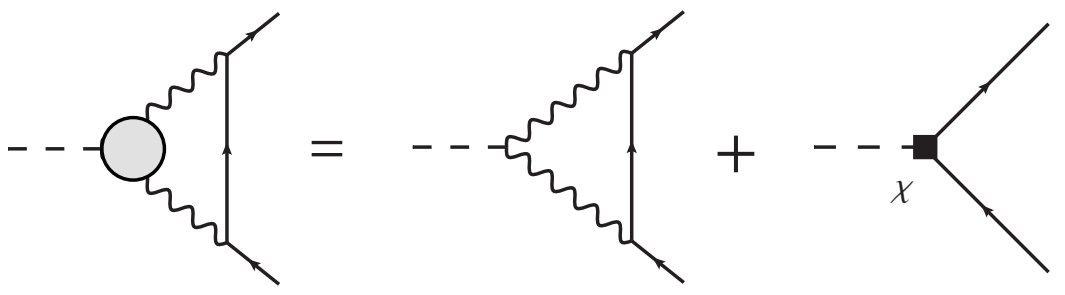

Figure 1: Leading-order contribution in the QED expansion (on the left) and its representation in terms of the leading order of the chiral perturbation theory (on the right).

Pions are complicated composite objects and their interactions are thus not point-like. This complexity is in the case of the two-photon decay described by a pion transition form factor $\mathcal{F}_{\pi^{0} \gamma^{*} \gamma^{*}}$, i.e. by the three-point correlator between a neutral pion and two electromagnetic currents. This important quantity enters not only the rare decay under discussion, but also the hadronic light-by-light scattering contribution to the already mentioned gyromagnetic ratio of the muon. For the rare decay, the leading-order (LO) contribution in the QED expansion is already a triangle loop diagram, which is depicted as the left hand side of the graphical equation in Fig. 1. It can be further represented within the Chiral Perturbation Theory $(\chi \mathrm{PT})$, where, in the LO, the complete form factor is replaced by the contact interaction given by the chiral anomaly. To get the UV-finite result, one has to add a counter term proportional to the coupling $\chi$ (see [3] for the complete Lagrangian), the finite part of which, $\chi^{(\mathrm{r})}(\mu)$, is a free parameter determined by this matching; see right hand side of the graphical equation in Fig. 1. This parameter is unique for any form factor and can be as well assigned to the measurements, which makes it a suitable tool for comparing the results among different theoretical approaches and eventual experiments. In case of the $\mathrm{KTeV}$ result, together with the Dorokhov approach [2] we find $\chi^{(\mathrm{r})}\left(M_{\rho}\right)=6.0 \pm 1.0$.

\section{Rare decay $\pi^{0} \rightarrow e^{+} e^{-}$and radiative corrections}

The pure-QED two-loop virtual radiative corrections were calculated in [4]. Since the compensation of the IR divergences is necessary, also the exact bremsstrahlung contributions were additionally taken into account in $[5,6]$. The overall size of the corrections was found to be

$$
\delta(0.95) \equiv \delta^{\mathrm{virt} .}+\delta^{\mathrm{BS}}(0.95)=(-5.5 \pm 0.2) \%,
$$

which differs significantly from the previous approximate calculations done in [7] and [8], where for $\delta(0.95)$ the authors obtained $-13.8 \%$ and $-13.3 \%$, respectively. Due to its weak dependence on $\chi^{(\mathrm{r})}$ it can be considered model-independent. After taking into account a proper calculation of the radiative corrections in the QED sector, the original discrepancy between SM and the KTeV experiment reduces to the $2 \sigma$ level or less, which can be expressed also effectively in terms of $\chi_{\mathrm{KTeV}}^{(\mathrm{r})}\left(M_{\rho}\right)=4.5 \pm 1.0$. To compare this result with some theoretical model, we present the value given by the Lowest-Meson Dominance (LMD) model $\chi_{\mathrm{LMD}}^{(\mathrm{r})}\left(M_{\rho}\right)=2.2 \pm 0.9$ [9]. In what follows we would like to check if using a more sophisticated model for the pion transition form factor $\mathcal{F}_{\pi^{0} \gamma^{*} \gamma^{*}}$ helps the current situation. 


\section{Two-Hadron Saturation for the $P V V$ correlator}

Driven by the remaining discrepancy in the rare pion decay, we investigated a possibility of getting an improved model for the pion transition form factor $\mathcal{F}_{\pi^{0} \gamma^{*} \gamma^{*}}\left(p^{2}, q^{2}\right)$. It would be an eligible alternative to the models available on the market and used by the community so far in terms of uncertainty treatment and fulfillment of requirements from various inputs. Within a family of large- $N_{c}$ motivated resonance-saturation models for the $P V V$ correlator in the chiral limit, we consider the following ansatz [10]:

$$
\Pi^{\mathrm{THS}}\left(r^{2} ; p^{2}, q^{2}\right)=\frac{B_{0} F^{2}}{r^{2}\left(r^{2}-M_{P}^{2}\right)} \frac{P\left(r^{2} ; p^{2}, q^{2}\right)}{\left(p^{2}-M_{V_{1}}^{2}\right)\left(p^{2}-M_{V_{2}}^{2}\right)\left(q^{2}-M_{V_{1}}^{2}\right)\left(q^{2}-M_{V_{2}}^{2}\right)} .
$$

We have taken into account two multiplets in both vector and pseudoscalar channels. To our knowledge such an approach was not used in the literature before. Together with the constraints we apply further, we call this approach the Two-Hadron Saturation (THS). In the numerator there stands a general polynomial which is symmetric in $p^{2}$ and $q^{2}$. Since the correlator must drop at large momenta, we can, in the very beginning, restrict the number of free parameters to 22 , so the polynomial looks as follows:

$$
P\left(r^{2} ; p^{2}, q^{2}\right)=c_{0} p^{2} q^{2}+c_{1}\left[\left(p^{2}\right)^{3} q^{2}+\left(q^{2}\right)^{3} p^{2}\right]+c_{2}\left(r^{2}\right)^{2} p^{2} q^{2}+\ldots
$$

To minimize the number of unknown parameters, we apply high- as well as low-energy theoretical constraints. First we demand satisfying of the leading-order OPE constraints [11] by our ansatz:

$$
\begin{gathered}
\Pi\left((\lambda r)^{2} ;(\lambda p)^{2},(\lambda q)^{2}\right)=\frac{1}{2} B_{0} F^{2} \frac{1}{\lambda^{4}} \frac{r^{2}+p^{2}+q^{2}}{r^{2} p^{2} q^{2}}+O\left(\frac{1}{\lambda^{6}}\right), \\
\Pi\left(r^{2} ;(\lambda p)^{2},(r-\lambda p)^{2}\right)=B_{0} F^{2} \frac{1}{\lambda^{2}} \frac{1}{r^{2} p^{2}}+O\left(\frac{1}{\lambda^{3}}\right) .
\end{gathered}
$$

It turns out that the third OPE constraint [11]

$$
\Pi\left((q+\lambda p)^{2} ;(\lambda p)^{2}, q^{2}\right)=\frac{1}{\lambda^{2}} \frac{1}{p^{2}} f\left(q^{2}\right)+O\left(\frac{1}{\lambda^{3}}\right),
$$

is automatically fulfilled by our ansatz. Here $f$ denotes a function (actually a two-point correlator [11]) that depends only on $q^{2}$ and not on the other kinematic variables $p^{2}$ and $p \cdot q$. Next, we define the $\pi V V$ correlator:

$$
\mathcal{F}_{\pi V V}\left(p^{2}, q^{2}\right) \equiv \frac{1}{\mathcal{Z}_{\pi}} \lim _{r^{2} \rightarrow 0} r^{2} \Pi\left(r^{2} ; p^{2}, q^{2}\right)
$$

where

$$
\mathcal{Z}_{\pi} \equiv \frac{i}{2}\left\langle 0\left|\left(\bar{u} \gamma_{5} u-\bar{d} \gamma_{5} d\right)\right| \pi^{0}\right\rangle=B_{0} F
$$

denotes the overlap between the pion field and the pseudoscalar quark density. Instead of involving subleading orders in the high-energy expansion we in addition apply the Brodsky-Lepage (B-L) constraint [12] on the resulting $\pi V V$ correlator:

$$
\frac{\mathcal{F}_{\pi V V}\left(0, q^{2}\right)}{\mathcal{F}_{\pi V V}(0,0)} \rightarrow-\frac{24 \pi^{2} F^{2}}{N_{c}} \frac{1}{q^{2}}, q^{2} \rightarrow-\infty
$$

Next, we define the pion transition form factor and match it at the photon point to the chiral anomaly:

$$
\mathcal{F}_{\pi^{0} \gamma^{*} \gamma^{*}}\left(p^{2}, q^{2}\right)=\frac{2}{3} \mathcal{F}_{\pi V V}\left(p^{2}, q^{2}\right), \quad \mathcal{F}_{\pi^{0} \gamma^{*} \gamma^{*}}(0,0)=-\frac{N_{c}}{12 \pi^{2} F} .
$$


Finally, we are left with a correlator that appears to have only one free dimensionless parameter $\kappa$ :

$$
\begin{aligned}
\mathcal{F}_{\pi V V}^{\mathrm{THS}}\left(p^{2}, q^{2}\right) & =-\frac{N_{c}}{8 \pi^{2} F} \frac{M_{V_{1}}^{4} M_{V_{2}}^{4}}{\left(p^{2}-M_{V_{1}}^{2}\right)\left(p^{2}-M_{V_{2}}^{2}\right)\left(q^{2}-M_{V_{1}}^{2}\right)\left(q^{2}-M_{V_{2}}^{2}\right)} \\
& \times\left\{1+\frac{\kappa}{2 N_{c}} \frac{p^{2} q^{2}}{(4 \pi F)^{4}}-\frac{4 \pi^{2} F^{2}\left(p^{2}+q^{2}\right)}{N_{c} M_{V_{1}}^{2} M_{V_{2}}^{2}}\left[6+\frac{p^{2} q^{2}}{M_{V_{1}}^{2} M_{V_{2}}^{2}}\right]\right\} .
\end{aligned}
$$

We determine the value of this parameter from a fit to the $\omega$ - $\pi$ transition form factor $\mathcal{F}_{\pi^{0} \omega \gamma^{*}}$ measurements [13] with the result $\kappa=21 \pm 3$. This choice stems from the fact that compared to $\rho$, the narrow $\omega$ resonance seems to be more suitable to resemble the large- $N_{c}$ world. Note that our result is independent of the mass $M_{P}$ of the first pseudoscalar excitation multiplet. This happens due to the fact that at the end of the day we could conveniently rescale the only free parameter left. For the mass of the first vector-meson multiplet $M_{V_{1}}$ we use the physical masses of $\rho$ or $\omega$ mesons. To account for the uncertainty given by taking only two multiples out of the whole tower of resonances, we vary the mass of the second multiplet $M_{V_{2}}$ within a range given by physical masses of the first and second vector-meson excitations, i.e.

$$
M_{V_{2}} \in[1400,1740] \mathrm{MeV} .
$$

In addition, we compare our results to similar approaches from the literature, namely to the VectorMeson Dominance (VMD) correlator [11, 14-16]

$$
\mathcal{F}_{\pi^{0} \gamma^{*} \gamma^{*}}^{\mathrm{VMD}}\left(p^{2}, q^{2}\right)=-\frac{N_{c}}{12 \pi^{2} F}\left[\frac{M_{V_{1}}^{4}}{\left(p^{2}-M_{V_{1}}^{2}\right)\left(q^{2}-M_{V_{1}}^{2}\right)}\right],
$$

which violates the OPE constraint (6), i.e. $\mathcal{F}_{\pi^{0} \gamma^{*} \gamma^{*}}^{\mathrm{VMD}}\left(q^{2}, q^{2}\right) \nsucc 1 / q^{2}, q^{2} \rightarrow-\infty$, or to the Lowest-Meson Dominance (LMD) model,

$$
\mathcal{F}_{\pi^{0} \gamma^{*} \gamma^{*}}^{\mathrm{LMD}}\left(p^{2}, q^{2}\right)=\mathcal{F}_{\pi^{0} \gamma^{*} \gamma^{*}}^{\mathrm{VMD}}\left(p^{2}, q^{2}\right)\left\{1-\frac{4 \pi^{2} F^{2}\left(p^{2}+q^{2}\right)}{N_{c} M_{V_{1}}^{4}}\right\},
$$

which strongly violates the B-L limit, i.e. $\mathcal{F}_{\pi^{0} \gamma^{*} \gamma^{*}}^{\mathrm{LMD}}\left(0, q^{2}\right) \ngtr 1 / q^{2}, q^{2} \rightarrow-\infty$. It is worth noting that in the singly off-shell regime, the correlator does not depend on $\kappa$. Thus one has a full predictive power for the associated quantities like the form factor slope $a_{\pi}$.

By construction the THS correlator satisfies all the mentioned constraints, but it is interesting to see how fast or slow the asymptotic limits are reached. This is shown in Fig. 2 where we display the singly virtual pion transition form factor (times the virtuality $q^{2}$ ) instead of the $\pi V V$ correlator. Note though that the relation (11) between the pion transition form factor and the $\pi V V$ correlator only amounts simply to a rescaling. In the plot we see a very narrow gray band and data. We first discuss the gray band, which nearly resembles a full line since its width is so small. The largest uncertainty comes from a variation of $M_{V_{2}}$ according to (13). This variation does not lead to very different results, which causes the curves to nearly collapse to one single line. Thus THS has rather high predictive power for the singly virtual pion transition form factor. We see that it reaches the asymptotic B-L limit rather early. We regard this intriguing behavior of our correlator as one of the highlights of our work.

Finally, we compare the THS result for the singly virtual pion transition form factor to VMD and LMD and to data. The LMD result for $\mathcal{F}_{\pi^{0} \gamma^{*} \gamma^{*}}\left(0, q^{2}\right)$ behaves as a constant for large $\left|q^{2}\right|$. Thus, it cannot even qualitatively explain the data since, of course, it diverges after being multiplied by $q^{2}$ (see the dash-dotted line). The results for VMD (dashed line) and THS are fairly close. CLEO data [17] 


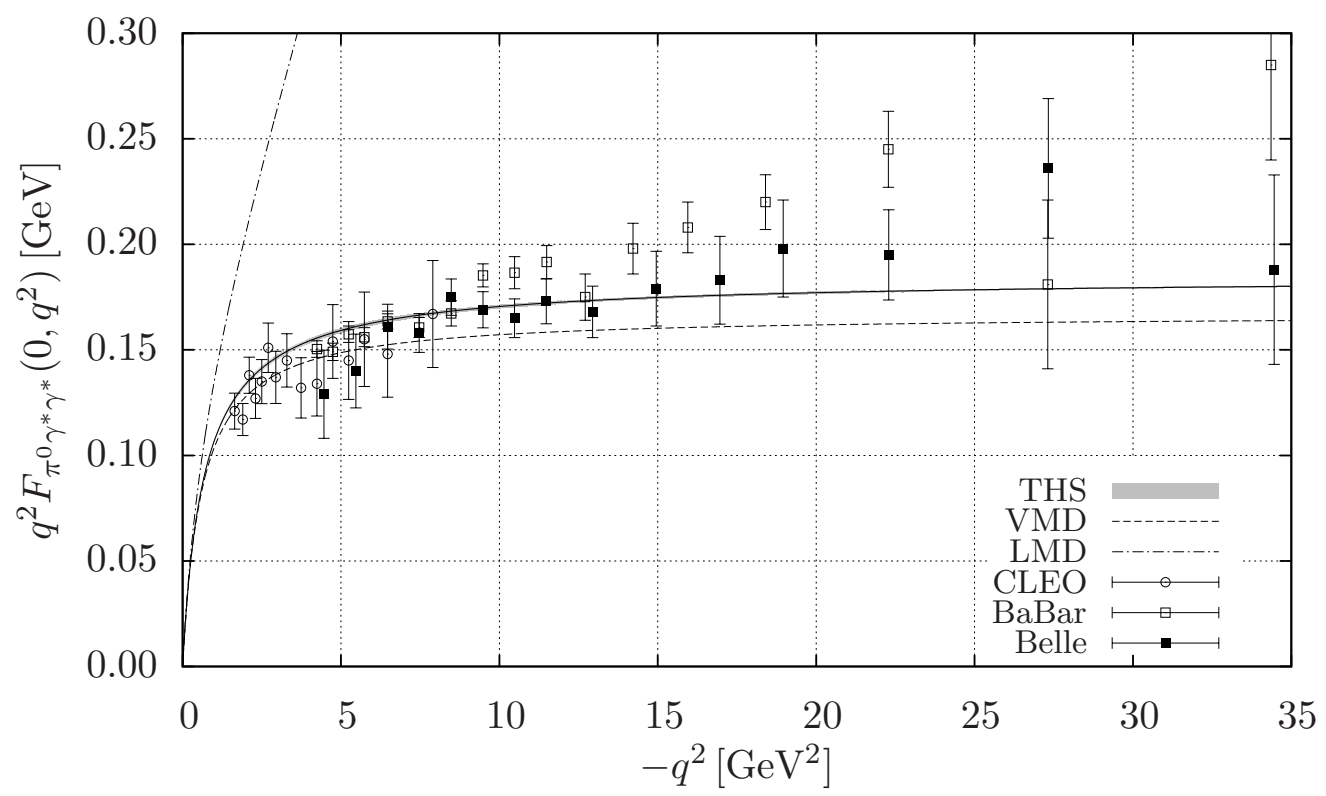

Figure 2: Singly virtual pion transition form factor as a function of the virtuality in the space-like region. The thin gray band constitutes the final THS prediction. See the main text for details how the uncertainties are determined. The corresponding curve that constitutes the center of the gray band has been made bold. The results from VMD and LMD are given by the dashed and dash-dotted lines, respectively. Data are taken from [17-19].

are only available for low virtualities $\left(-q^{2}<8 \mathrm{GeV}^{2}\right)$ and prefer VMD to some extent though THS appears to be acceptable as well. The situation turns around for higher virtualities. THS describes the Belle data [19] better than VMD. The early onset of the asymptotic behavior of the THS result for the singly virtual pion transition form factor makes THS essentially identical to the B-L limit. Not surprisingly then, THS agrees well with the Belle data and is at odds with the BaBar data [18]. In view of these complications we have decided to stick to the B-L constraint (10) right from the start. We do not attempt to contribute to a clarification of the differences between Belle and BaBar.

\section{Results}

Taking into account the complete set of the next-to-leading-order (NLO) radiative corrections presented in Section 3 and the THS model presented in the previous section, we get for the theoretical prediction of the quantity measured by $\mathrm{KTeV}$ experiment $B^{\mathrm{THS}}=(5.8 \pm 0.2) \times 10^{-8}$. If we recall the experimental value $B^{\mathrm{KTeV}}=(6.44 \pm 0.33) \times 10^{-8}$, we find that the disagreement is now at the level of only $1.8 \sigma$. The matching on the LO $\chi$ PT gives $\chi_{\mathrm{THS}}^{(\mathrm{r})}\left(M_{\rho}\right)=2.2 \pm 0.7$. Thus, if the KTeV result will be confirmed by future experiments, two scenarios are conceivable: a) either there are indeed some aspects of the THS approach which are not well-suited for the process $\pi^{0} \rightarrow e^{+} e^{-}$, or b) the beyond-Standard Model physics influences the rare pion decay significantly. But under the present circumstances the current discrepancy is inconclusive. Actually, the quantity which was originally 


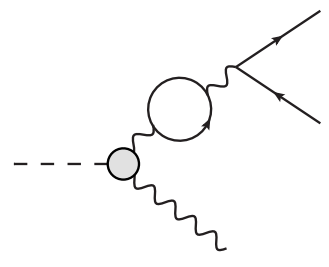

(a)

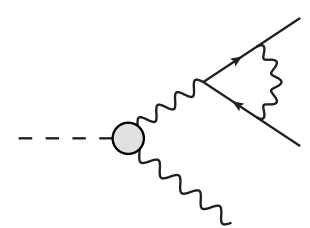

(b)

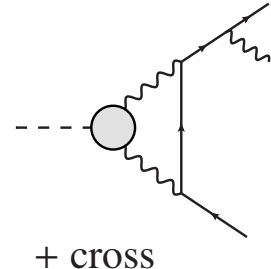

(c)

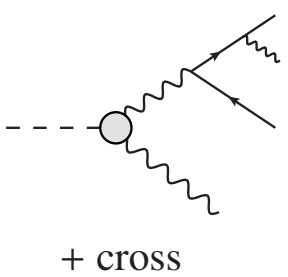

(d)

Figure 3: Radiative corrections to the Dalitz decay $\pi^{0} \rightarrow e^{+} e^{-} \gamma$ : (a) vacuum polarization insertion, (b) correction to the QED vertex, (c) one-loop one-photon irreducible contribution and (d) bremsstrahlung. Note that in (c), 'cross' stands for additional two diagrams with the photon coming from the positron as well as the inner (the box diagram) fermion lines. In (d), 'cross' then stands for the diagram where the bremsstrahlung photon is radiated by the positron as well as for the other two diagrams where the two photons in the final state are interchanged.

measured by $\mathrm{KTeV}$ was a ratio of branching ratios, where the Dalitz decay was used as the normalization channel.

$$
\left.\frac{\Gamma\left(\pi^{0} \rightarrow e^{+} e^{-}(\gamma), x>0.95\right)}{\Gamma\left(\pi^{0} \rightarrow e^{+} e^{-} \gamma(\gamma), x>0.2319\right)}\right|_{\mathrm{KTeV}}=(1.685 \pm 0.064 \pm 0.027) \times 10^{-4} .
$$

This is also why it is important to have the latest mentioned process under control. We will discuss it in the next section.

\section{Dalitz decay of a neutral pion}

The second most important decay channel of a neutral pion, right after the process $\pi^{0} \rightarrow \gamma \gamma$, is the Dalitz decay $\pi^{0} \rightarrow e^{+} e^{-} \gamma$ with a branching ratio (1.174 \pm 0.035$) \%$ [20]. This decay was first studied by Richard H. Dalitz [21], whose name it carries. The experimental data of this process provide the information about the singly off-shell pion transition form factor $\mathcal{F}_{\pi^{0} \gamma^{*} \gamma^{*}}\left(0, q^{2}\right)$ in the time-like region and in particular about its slope parameter $a_{\pi}$.

Radiative corrections to the total decay rate of the Dalitz decay were first addressed by D. Joseph [22]. The pioneering study of the corrections to the differential decay rate was done by B. E. Lautrup and J. Smith [23] using the soft-photon approximation. Soon after, this analysis was extended by K. O. Mikaelian and J. Smith [24] by the hard-photon corrections to the whole range of the bremsstrahlung photon energy. Let us also mention that a systematic treatment of the NLO corrections to the Dalitz decay of the neutral pion in the framework of the Chiral Perturbation Theory with dynamical leptons and photons was studied in [25].

The table of the radiative corrections $\delta(x, y)$ to the leading-order differential decay rate was presented as one of the main results of the above mentioned classical work of Mikaelian and Smith [24]. Such a table would be very useful for the Monte Carlo simulations in experiments covering $\pi^{0}$ decays, for instance the NA48 experiment at CERN [26]. In practice, for the table of values $\delta(x, y)$, which was published in [24], an interpolation or extrapolation procedure needs to be used in order to get the radiative correction at any desired point of the Dalitz plot. This might lead to a large uncertainty. We have therefore recalculated, generalized and extended [27] the results presented in [24]; see Fig. 3 
for the diagram representation of the contributions which where considered. A C++ code which returns the correction for any given pair of kinematic variables $x$ and $y$ over the whole physical region was developed. This implementation of the calculations became a part of the simulation software of the NA62 experiment and is without any doubt an important component of the current experimental analysis.

It is worth emphasizing that we have included in our approach the one-loop one-photon irreducible contribution (see Fig. 3c), which was considered to be negligible in the original paper [24] due to its proportionality to the lepton mass. This statement had been corrected in [28] many years before the debate about this issue was closed; see e.g. [29, 30]. Especially for a large invariant dilepton mass, this correction is indeed important and changes significantly the values of entries stated in Table I of [24]. The complete set of the NLO QED radiative corrections is thus now available. As we have not neglected the higher order terms in the electron mass and included also the muon loop contribution to the vacuum polarization insertion correction, our results can be in principle also applied to the other related processes. The decay of the $\eta^{(\prime)}$ meson to a muon pair and a photon, i.e. the process $\eta^{(\prime)} \rightarrow \ell^{+} \ell^{-} \gamma$, where the masses of the final state particles are not anymore negligible in comparison to the decaying pseudoscalar, is such an example. On the other hand, when $\eta^{(\prime)}$ meson and its decays come into play, some peculiarities inevitably appear: additional corrections need to be taken into account and $\eta-\eta^{\prime}$ mixing should be properly incorporated.

\section{Conclusions}

The complete set of the NLO radiative corrections in the QED sector for both discussed processes, the rare decay of a neutral pion and the Dalitz decay, is now available. In the case of the process $\pi^{0} \rightarrow e^{+} e^{-}$, the important contribution of the analytical two-loop QED corrections together with the bremsstrahlung contribution beyond the soft-photon approximation were reviewed. The overall value of the correction substantially differs from the previous approximative estimations. Since these were used in the experimental analysis of the $\mathrm{KTeV}$ experiment, taking into account the exact result reduces the discrepancy significantly down to $2 \sigma$. We have thus showed on the case of the KTeV experiment that incorporating these updated calculations in future experimental analyses should be essential. The similar statement than holds for the Dalitz decay and the experiments which deal with the related processes, i.e. the decays of mesons to a lepton pair and a photon. We have updated the classical work of Mikaelian and Smith most importantly by adding the one-photon irreducible contribution. Since the generality was preserved during the calculation, this fact allows us to use the achieved results for the $\eta^{(\prime)}$ decays of the same type. The work summarizing all the necessary contributions and subtleties in these cases is close to completion. Last but not least, we also presented the THS model for the electromagnetic transition form factor $\mathcal{F}_{\pi^{0} \gamma^{*} \gamma^{*}}\left(p^{2}, q^{2}\right)$, which satisfies all main theoretical constraints which follow from the QCD. It is phenomenologically very successful and for a wide range of quantities it gives reliable predictions which are consistent with the experimental data. In the singly virtual regime, the THS model is compatible with the recent Belle data. Taking all the pieces together, we get a reasonable SM prediction for the pion rare decay branching ratio, which differs from the $\mathrm{KTeV}$ result by inconclusive $1.8 \sigma$.

\section{Acknowledgements}

This contribution is based on the collaboration with K. Kampf, J. Novotný (Charles University) and S. Leupold (Uppsala University). It was supported by the Charles University (grant GAUK 700214) and by the Czech Science Foundation (grant GAČR 15-18080S). 


\section{References}

[1] E. Abouzaid et al. (KTeV), Phys. Rev. D75, 012004 (2007)

[2] A.E. Dorokhov, M.A. Ivanov, Phys. Rev. D75, 114007 (2007)

[3] M.J. Savage, M.E. Luke, M.B. Wise, Phys. Lett. B291, 481 (1992)

[4] P. Vaško, J. Novotný, JHEP 1110, 122 (2011)

[5] T. Husek, K. Kampf, J. Novotný, Eur. Phys. J. C74, 3010 (2014)

[6] T. Husek, K. Kampf, J. Novotný, AIP Conf. Proc. 1701, 040008 (2016)

[7] Bergström, L., Z. Phys. C20, 135 (1983)

[8] A. Dorokhov, E. Kuraev, Y. Bystritskiy, M. Secansky, Eur. Phys. J. C55, 193 (2008)

[9] M. Knecht, S. Peris, M. Perrottet, E. de Rafael, Phys. Rev. Lett. 83, 5230 (1999)

[10] T. Husek, S. Leupold, Eur. Phys. J. C75, 586 (2015)

[11] M. Knecht, A. Nyffeler, Eur. Phys. J. C21, 659 (2001)

[12] S.J. Brodsky, G.P. Lepage, Phys. Rev. D24, 1808 (1981)

[13] R. Arnaldi et al. (NA60), Phys. Lett. B677, 260 (2009)

[14] J.J. Sakurai, Currents and Mesons (University of Chicago Press, Chicago, 1969)

[15] L. Landsberg, Phys. Rept. 128, 301 (1985)

[16] K. Kampf, J. Novotný, Phys. Rev. D84, 014036 (2011)

[17] J. Gronberg et al. (CLEO), Phys. Rev. D57, 33 (1998)

[18] B. Aubert et al. (BaBar), Phys. Rev. D80, 052002 (2009)

[19] S. Uehara et al. (Belle), Phys. Rev. D86, 092007 (2012)

[20] K. Olive et al. (Particle Data Group), Chin. Phys. C38, 090001 (2014)

[21] R. Dalitz, Proc. Phys. Soc. A64, 667 (1951)

[22] D. Joseph, Nuovo Cimento 16, 997 (1960)

[23] B. Lautrup, J. Smith, Phys. Rev. D3, 1122 (1971)

[24] K. Mikaelian, J. Smith, Phys. Rev. D5, 1763 (1972)

[25] K. Kampf, M. Knecht, J. Novotný, Eur. Phys. J. C46, 191 (2006)

[26] J.R. Batley et al. (NA48/2), Phys. Lett. B746, 178 (2015)

[27] T. Husek, K. Kampf, J. Novotný, Phys. Rev. D92, 054027 (2015)

[28] G.B. Tupper, T.R. Grose, M.A. Samuel, Phys. Rev. D28, 2905 (1983)

[29] M. Lambin, J. Pestieau, Phys. Rev. D31, 211 (1985)

[30] G. Tupper, Phys. Rev. D35, 1726 (1987) 